Die Erstausgabe der Schweizerischen Ärztezeitung erschien 1920 und war fortan dreisprachig angeschrieben. Seit 1938 ist Rätoromanisch als vierte Landessprache in der Verfassung verankert und seit 1996 auch Amtssprache im Behördenverkehr. Auf Antrag des Bündner Ärztevereins ist die Ärztezeitung ab heute viersprachig angeschrieben. Gratulaziuns fitg cordialas a tut ils medis da la Rumantschia! Nus ans legrain cun vus. In pitschen giavisch da mai: publitgar da temp en temp in artitgel rumantsch en la Gasetta dals medis svizzers - quai fiss vairamain genial.

\title{
Rumantsch - la quarta lingua naziunala Romanisch - die vierte Landessprache
}

\author{
Claude Leissing \\ Dr. med., President da I'Uniun grischuna da medis; Präsident des Bündner Ärztevereins
}

Cun questa ediziun dal Gasetta dals medis svizzers cumenza ina nova èra: a partir dad immediat è represchentada sin il frontispizi dal meritaivel carnet mellen era la lingua rumantscha: Gasetta dals medis svizzers. Tgi sa, forsa cumpara en l'avegnir da temp en temp schizunt in pitschen artitgel per rumantsch.

Tenor la glista dals medis www.doctorfmh.ch chapeschan en total 92 medias e medis che pratitgeschan en Svizra rumantsch, 35 dad els lavuran en il Grischun. 40 medias e medis da chasa inditgeschan dad era discurrer rumantsch, 21 dad els lavuran en il Grischun. Sco quai che nus avain constatà han numerusas collegas e numerus collegas che discurran rumantsch anc betg profità da l'occasiun dad inditgar lur enconuschientschas da la lingua era sin www.doctorfmh.ch. Nus lain intimar las collegas ed ils collegas dad examinar lur endataziuns e da cumplettar quellas via myFMH.

La populaziun rumantscha vegn pli e pli pitschna. Gia baud èn ins sfurzads da bandunar per il studi e la scolaziun la regiun d'origin e dad ir en autras parts da la Svizra. Igl è normal che persunas ch'emigreschan en autras regiuns linguisticas na dovran plaun a plaun betg pli lur lingua originara. Dapi il 1990 è la cumpart da la populaziun svizra ch'inditgescha il rumantsch sco meglra lingua sa sbassada adina pli fitg sin oz damain che $14,9 \%$ en il chantun Grischun e pli pauc che $0,3 \%$ en ils chantuns da la Svizra Bassa. A quest trend vulan las Rumantschas ed ils Rumantschs far frunt. Cun menziunar la quarta lingua naziunala sin il frontispizi dal Gasetta dals medis svizzers vulain nus sco Uniun grischuna da medis metter, ensemen cun la Federaziun dals medis svizzers FMH, in signal per la multifariadad da nossa Svizra e surtut era per la multifariadad da las medias e dals medis.
Mit dieser Ausgabe der Schweizerischen Ärztezeitung beginnt eine neue Ära: Ab sofort ist auf der Titelseite des altgedienten gelben Heftlis auch die romanische Sprache vertreten: Gasetta dals medis svizzers. Wer weiss, vielleicht wird in Zukunft sogar hie und da ein kleiner Artikel in rätoromanischer Sprache zu finden sein.

Gemäss dem Ärzteverzeichnis www.doctorfmh.ch beherrschen insgesamt 92 in der Schweiz tätige Ärztinnen und Ärzte die romanische Sprache, 35 davon sind in Graubünden tätig. 40 Hausärzte geben an, auch Romanisch zu sprechen, 21 davon arbeiten wiederum in Graubünden. Wie wir feststellen konnten, haben zahlreiche Romanisch sprechende Kolleginnen und Kollegen die Chance noch nicht genutzt, ihre Sprachkenntnisse auch auf www.doctorfmh.ch festzuhalten. Wir möchten diese auffordern, ihre Angaben zu überprüfen und über myFMH zu ergänzen.

Die Romanisch sprechende Bevölkerung schwindet zunehmend. Schon früh ist man gezwungen, für Studium und Ausbildung die Stammlande zu verlassen und sich in andere Teile der Schweiz zu begeben. Es ist der normale Lauf der Dinge, dass bei Personen, die in andere Sprachregionen abwandern, die ursprüngliche Sprache allmählich ausser Gebrauch gerät. Seit 1990 ist der Anteil der Bevölkerung der Schweiz, die das Romanische als bestbeherrschte Sprache nennt, immer weiter auf heute weniger als 14,9\% im Kanton Graubünden und weniger als 0,3\% in den Kantonen des Mittellandes gesunken. Diesem Trend wollen die Romanen entgegenwirken. Mit der Erwähnung der vierten Landessprache auf der Titelseite der Schweizerischen Ärztezeitung wollen wir als Bündner Ärzteverein gemeinsam mit der FMH ein Zeichen setzen für die Vielfalt unserer Schweiz und insbesondere auch für die Vielfalt der Ärzteschaft. 